\title{
Gas Chromatographic Analysis of Mono-, Di-, and Tripentaerythritol in the Form of Their Trimethylsilyl Ethers*
}

\author{
BENGT SMITH and LENNART TULLBERG \\ Institutionen för Organisk Kemi, Chalmers Tekniska Högskola, Göteborg, Sweden
}

\begin{abstract}
The mixture of mono-, di-, and tripentaerythritol, present in commercial pentaerythritol, may be analysed by converting the three polyols into their trimethylsilyl ethers which are volatile enough to be separated by gas chromatography.
\end{abstract}

$\mathbf{P}$ entaerythritol is one of the more important technical polyols manufactured and, accordingly, it is of great interest to be able to analyse the product with sufficient accuracy. The determination of the main components in pentaerythritol, mono-, di-, and tripentaerythritol, $* * \quad\left(\mathrm{HOCH}_{2}\right)_{4} \mathrm{C}$, $\left[\left(\mathrm{HOCH}_{2}\right)_{3} \mathrm{CCH}_{2}\right]_{2} \mathrm{O}$, and $\left[\left(\mathrm{HOCH}_{2}\right)_{3} \mathrm{CCH}_{2} \mathrm{OCH}_{2}\right]_{2} \mathrm{C}\left(\mathrm{CH}_{2} \mathrm{OH}\right)_{2}$ has, however, met with considerable difficulties.

For a long time the only method available for the assay of pentaerythritol was that of Kraft ${ }^{1}$ involving the precipitation of MPE in the form of its dibenzylidene acetal by reaction with benzaldehyde. The weak point of the method is that the results are influenced by the presence of DPE in concentrations higher than $2 \%$. Also other impurities present in crude pentaerythritol may cause erroneous results. ${ }^{2}$ The purest grade of MPE sold commercially contains about 2 to $3 \%$ DPE. While the dibenzylidene acetal method can be used for the analysis of this product it is clearly unsuitable for analysing various mixtures of MPE and DPE, containing from 10 to $30 \% \mathrm{DPE}$, which are also on the market. Sporek and Williams ${ }^{2}$ tried to remedy the deficiencies in the method by combining it with an adsorption chromatographic procedure for the separation of MPE in a state pure enough to be successfully assayed according to Kraft.

Wyler ${ }^{3}$ developed a method for the determination of DPE in mixtures with MPE based on the precipitation of the former compound from aqueous

\footnotetext{
* The main part of this work was completed already by mid of 1961, but has been withheld from publication on request of the company for which the investigation was made. The original work has recently been completed with some experiments using programmed temperature gas chromatography.

** In the following the abbreviations MPE, DPE and TPE will be used.
} 
solutions containing both of them. For good results the specified conditions must be strictly adhered to and, moreover, higher molecular weight pentaerythritols and pentaerythritol formals were found to interfere. An infrared method has been reported by Jaffe and Pinchas ${ }^{4}$ for the determination of DPE. It is based on the occurrence of a characteristic absorption band at $1115 \mathrm{~cm}^{-1}$ from the ether linkage present in DPE, but absent in MPE. Because of the low solubility of the pentaerythritols in solvents suitable for infrared spectroscopic measurements, they have first to be converted to their acetates by treatment with acetic anhydride. Since TPE and higher pentaerythritols also contain ether linkages these polyols, if present, will be determined as DPE.

An X-ray diffraction method, claimed to be specific for MPE and DPE, was decribed by Carazzolo. 5 This method seems to give results of high accuracy, but the instrument necessary for the analysis is not likely to be available at laboratories working in this field.

Experiments with acetates and trimethylsilyl ethers of MPE and DPE using constant temperature gas chromatography

In the previous section the development of the pentaerythritol analysis up to 1960 , when we started our investigations, has been given. We turned our attention to a method which had not been tried previously but which could be anticipated to have good prospects in this field, namely gas chromatography. Although it was obvious that the polyols in question were insufficiently volatile and too unstable to be separated directly, there was the possibility of converting them into more volatile and stable derivatives e.g. esters or ethers.

Acetates. The acetates were nearest to hand. MPE tetraacetate and DPE hexaacetate were prepared from the corresponding polyols and acetic anhydride. The boiling points were for MPE tetraacetate about $325^{\circ} \mathrm{C}$ at atmospheric pressure and for DPE hexaacetate about $240^{\circ} \mathrm{C}$ at $1 \mathrm{~mm}$. The yields were $90 \%$ or higher. Since unavoidable losses are made in the syntheses it appears that the yield is quantitative or nearly so. Herewith the first requirement for a quantitative analysis was fulfilled. The second involved that the acetates were volatile and stable enough to be separated by gas chromatography. With the instrument available and using isothermal operation at a maximum temperature of $225^{\circ} \mathrm{C}$ this turned out to be the case for MPE tetraacetate but not for DPE hexaacetate. The latter compound certainly passed through the chromatograph but the shape of the peak made it unsuitable for quantitative analysis. As seen from Fig. 1 the peak given by the MPE tetraacetate was quite satisfactory. Although a quantitative analysis of MPE is undoubtedly possible, we did not study the acetate method further since we also wanted to analyze for DPE.* Instead we turned our attention to the ether derivatives of the pentaerythritols.

\footnotetext{
* Since this work was completed Wiersma et al. ${ }^{6}$ have determined MPE, DPE and TPE quantitatively after acetylation using programmed temperature gas chromatography in the range 220 to $320^{\circ} \mathrm{C}$. In the same way Esposito and Swann ${ }^{7}$ identified MPE liberated on decomposition of synthetic resins.
} 


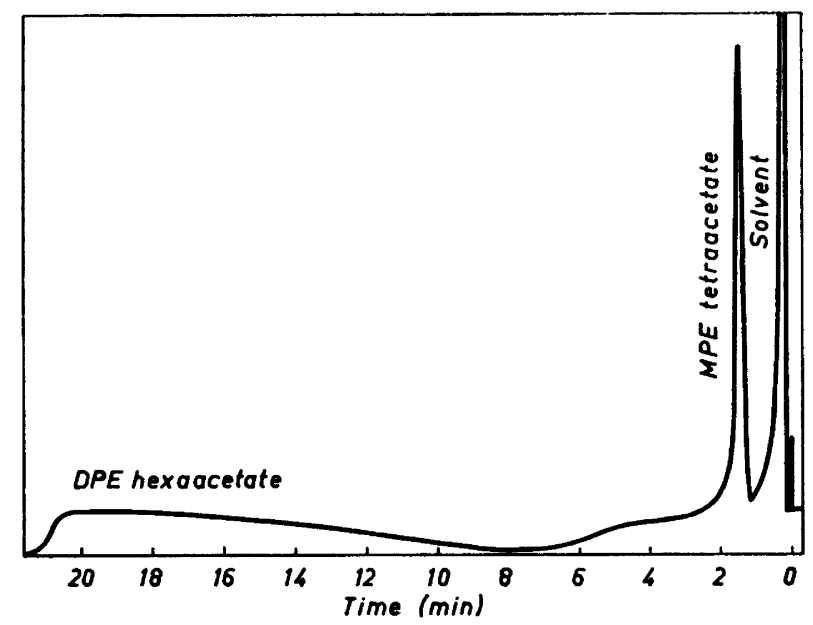

Fig. 1. Separation of acetates of MPE and DPE. Column: Apiezon M, $1 \mathrm{~m} \times 4 \mathrm{~mm}$. Temperature $225^{\circ} \mathrm{C}$, flow of helium $80 \mathrm{ml} / \mathrm{min}$.

Trimethylsilyl ethers. There is no simple method for preparing ethers like the methyl and ethyl ethers from pentaerythritols and, moreover, the yields obtained are generally low. However, ethers which can be prepared in a simple way and in high yield are the trimethylsilyl ethers. The ethers of MPE and DPE were synthezised by refluxing the polyols with trimethylchlorosilane in pyridine. The etherification takes place according to the equation

$$
\equiv \mathrm{C}-\mathrm{OH}+\left(\mathrm{CH}_{3}\right)_{3} \mathrm{SiCl}+\mathrm{C}_{5} \mathrm{H}_{5} \mathrm{~N} \rightarrow \equiv \mathrm{C}-\mathrm{O}-\mathrm{Si}\left(\mathrm{CH}_{3}\right)_{3}+\mathrm{C}_{5} \mathrm{H}_{5} \mathrm{~N}, \mathrm{HCl}
$$

The boiling point of the MPE tetra(trimethylsilyl)ether was about $150^{\circ} \mathrm{C}$ at $17 \mathrm{~mm}$ and that of the DPE hexa(trimethylsilyl) ether about $205^{\circ} \mathrm{C}$ at 10 $\mathrm{mm}$. Accordingly, the boiling points of the trimethylsilyl ethers of MPE and DPE are appreciably lower than the boiling points of the corresponding acetates (cf. above). The infrared spectra of the products obtained revealed, however, the presence of free hydroxyl groups. Accordingly, the trimethylsilylation was not complete under the conditions used. Only by performing the reaction in a sealed tube at $150^{\circ} \mathrm{C}$ could a complete reaction be achieved.

The trimethylsilyl ethers of MPE and DPE could be separated at the maximum temperature of the instrument available $\left(225^{\circ} \mathrm{C}\right)$ and gave peaks suitable for quantitative evaluation (see Fig. 2). Attempts to run the TPE octa(trimethylsilyl) ether were not so successful since the peak obtained was very flat.

The quantitative aspects were investigated using the internal standard method. For the determination of the MPE ether, diphenyl was used as internal standard and the peak heights were compared and related to the amounts. Fluorene was the internal standard used for the DPE ether and in this case the areas under the peaks were compared (see Fig. 2). The necessity of using

Acta Chem. Scand. 19 (1965) No. 3 
Table 1. Analysis values of MPE and DPE using constant temperature gas chromato. graphy.

\begin{tabular}{cccc}
$\begin{array}{c}\text { Experiment } \\
\text { No. }\end{array}$ & $\begin{array}{c}\text { True value } \\
(\mathrm{g})\end{array}$ & $\begin{array}{c}\text { Found } \\
(\mathrm{g})\end{array}$ & $\begin{array}{c}\text { Error } \\
\%\end{array}$ \\
\hline MPE-1 & 0.0507 & 0.0511 & +0.8 \\
MPE-2 & 0.0533 & 0.0529 & -0.8 \\
MPE-3 & 0.0536 & 0.0531 & -0.9 \\
MPE-4 & 0.0385 & 0.0378 & -1.8 \\
\hline DPE-1 & & & -3.3 \\
DPE-2 & 0.0240 & 0.0232 & +3.4 \\
DPE-3 & 0.0445 & 0.0460 & +0.8 \\
\hline
\end{tabular}

two internal standards is due to the dissimilarity in shape of the two peaks. Some representative quantitative values are given in Table 1 . As seen, MPE and DPE may be determined with good accuracy using this method.

Experiments with trimethylsilylethers of MPE, DPE and TPE using programmed temperature gas chromatography

The construction of dual column high-temperature program chromatographs has made possible the separation and determination of many high boiling compounds which were not analysable using conventional single column instruments with isothermal operation. Recently such an instrument became available at this laboratory. Since the problem of determining the components in crude pentaerythritol had not been completely solved in the foregoing, we

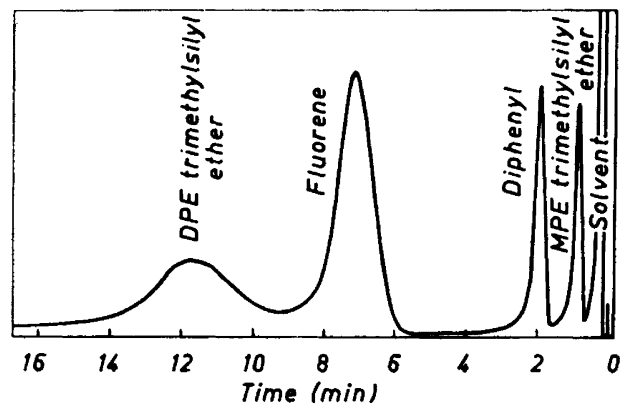

Fig. 2. Separation of trimethylsilyl ethers of MPE and DPE. Internal standards diphenyl and fluorene. Column. Apiezon $\mathrm{M}, 1 \mathrm{~m} \times 4 \mathrm{~mm}$. Temperature $225^{\circ} \mathrm{C}$, flow of helium $80 \mathrm{ml} / \mathrm{min}$.

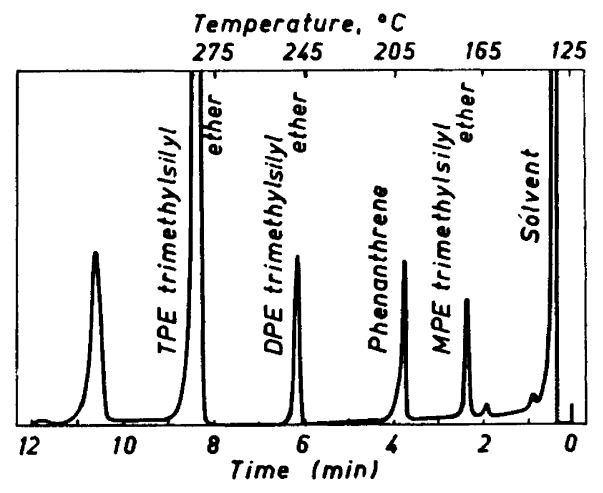

Fig. 3. Separation of trimethylsilyl ethers of MPE, DPE, and TPE obtained from crude TPE. Internal standard phenanthrene. Column: silicone gum SE-30, 6 feet $\times 1 / 8$ inch. Temperature programmed 125 to $275^{\circ} \mathrm{C}$, flow of nitrogen $25 \mathrm{ml} / \mathrm{min}$.

Acta Chem. Scand. 19 (1965) No. 3 
decided to investigate the possibilities of programmed temperature gas chromatography in the present case.

Fig. 3 shows a chromatogram from a separation of the trimethylsilyl ethers of MPE, DPE and TPE. There were no difficulties in eluting and separating the three compounds and excellent peaks were obtained, suitable for internal standard analysis by peak height comparison. A fourth peak of unknown origin also appeared. The compound responsible for this peak is present in crude TPE and may be tetrapentaerythritol. However, no authentic sample of this compound was available to prove the assumption. The results clearly indicate the possibility of analysing for pentaerythritols of higher molecular weight than TPE using the trimethylsilylation method.

One of the advantages of the present method in comparison with the acetate method $^{6}$ is the lower temperature range necessary for eluting the pentaerythritol derivatives. The maximum temperature used in the present case for the elution of the TPE octa(trimethylsilyl) ether was $275^{\circ}$ while about $300^{\circ}$ was necessary for the elution of the corresponding acetate. With the lower temperature follows an increased length of life of the stationary phase and the risk of thermal decomposition of the compounds being separated is less. A disadvantage, in comparison with the acetate method, is the somewhat more complicated procedure for the preparation of the trimethylsilyl ethers. However, the synthesis has now been simplified and presents no difficulties (see the experimental section, method 2). The necessary heating time is longer in the trimethylsilylation method than in the acetylation method, mainly because of the low solubilities of DPE and TPE. MPE, e.g., reacts rapidly on refluxing with a mixture of hexamethyldisilazane and trimethylchlorosilane for a couple of minutes. DPE, however, which is appreciably less soluble in the reagent mixture, has to be heated in a sealed tube for more than $3 \mathrm{~h}$ in order to ensure complete reaction. It might be that this reaction time could be decreased by adding suitable solvents.

To study the quantitative analysis of pentaerythritol trimethylsilyl ethers using programmed temperature operation, some experiments were made with MPE and DPE.* In this case only one internal standard, phenanthrene, was

Table 2. Analysis values of MPE and DPE using programmed temperature gas chromatography.

\begin{tabular}{cccc}
\hline $\begin{array}{c}\text { Experiment } \\
\text { No. }\end{array}$ & $\begin{array}{c}\text { True value } \\
(\mathrm{g})\end{array}$ & $\begin{array}{c}\text { Found } \\
(\mathrm{g})\end{array}$ & $\begin{array}{c}\text { Error } \\
\%\end{array}$ \\
\hline MPE-1 & 0.0698 & 0.0702 & +0.6 \\
MPE-2 & 0.0618 & 0.0614 & -0.6 \\
MPE-3 & 0.0434 & 0.0438 & +0.9 \\
\hline & & & 0 \\
DPE-1 & 0.0616 & 0.0616 & +2.0 \\
DPE-2 & 0.0394 & 0.0402 & -3.0 \\
\hline
\end{tabular}

* No experiments were made with TPE since no pure sample was available for calibration. Acta Chem. Scand. 19 (1965) No. 3 
necessary and the peak heights were compared and related to the amounts (see Fig. 3). Some results are given in Table 2. It is recommended when making quantitative analyses not to increase the temperature too rapidly since the reproducibility of the heating might be insufficient at high heating rates. In addition to MPE, DPE, and TPE, crude pentaerythritol may also contain small amounts of formals. We have not studied the analysis of these compounds but, since the formals in question contain free hydroxyls, ${ }^{6}$ they can undoubtedly be separated and determined as trimethylsilyl ethers.

\section{EXPERIMENTAL}

\section{Preparation of the trimethylsilyl ethers}

1. Using trimethylchlorosilane and pyridine. In our first experiments the silylation of the pentaerythritols was performed using trimethylchlorosilane and pyridine. Pyridine serves to bind the hydrogen chloride formed and also functions as a solvent. A detailed description of the etherification procedure has been given previously. ${ }^{8}$ It should only be mentioned here that, if oxygen is used instead of air in the blast lamp, no cooling of the test tube is necessary on sealing it. Heating to $150^{\circ}$ for $5 \mathrm{~h}$ is sufficient for complete conversion but we have generally chosen to heat the sealed tubes overnight $(10-15 \mathrm{~h})$. It is recommended to extract the precipitate of pyridine hydrochloride with benzene since the values obtained when no extraction is made tend to be erratic. The internal standard, diphenyl for the MPE ether and fluorene for the DPE ether, may be added at the beginning or after the extraction, but we have preferred to use the latter method.

2. Using hexamethyldisilazane and trimethylchlorosilane. This method is considered to be an improvement of the previous procedure. About $0.1 \mathrm{~g}$ of the pentaerythritol mixture was weighed in a dry pyrex test tube $(1 \times 10 \mathrm{~cm})$ together with the internal standard, phenanthrene. Hexamethyldisilazane and trimethylchlorosilane, $0.5 \mathrm{ml}$ of each, were then added and the tube sealed near the mouth using a blast lamp and oxygen/coal gas. The sealed tube was heated overnight $(10-15 \mathrm{~h})$ at $150^{\circ}$. The overnight heating was chosen for convenience. It seems that about $5 \mathrm{~h}$ are sufficient to ensure complete reaction of DPE and TPE. MPE reacts, as mentioned previously, very rapidly.

The cool ampoule was opened. Benzene $(0.75 \mathrm{ml})$ was added and the contents of the tube shaken in order to ensure complete solution of the trimethylsilyl ethers and the phenanthrene. The precipitate of ammonium chloride present was knocked down using a centrifuge and the clear solution chromatographed.

\section{Gas chromatographic separations}

Isothermal separations. For these separations a Perkin-Elmer Vapour Fractometer Model $116 \mathrm{E}$, equipped with a thermistor detector, was utilized. The column generally used was a $1 \mathrm{~m} \times 4 \mathrm{~mm}$ aluminium column containing Apiezon M (20 parts) on Celite $545,60-100$ mesh ( 80 parts). However, silicone oils or gums of sufficiently low volatility can also be used. The instrument was run at its maximum temperature, about $225^{\circ}$, the flow of helium being $80 \mathrm{ml} / \mathrm{min}$.

Programmed temperature separations were made using a Perkin-Elmer gas chromatograph Model 800. This instrument is a dual column temperature program chromatograph, equipped with a differential flame ionization detector. It may be operated at high temperatures with a minimum base line instability due to column substrate elution since, in the differential detector, a reference flame compensates for base line shift due to bleeding. Two $1 / 8$ inch $\times 6$ feet matched columns containing $1.5 \% \mathrm{SE}-30$ silicone gum on silanized Chromosorb W $(80-100 \mathrm{mesh}){ }^{*}$ were used for the separation. One of the columns functions as a reference column and is not involved in the separation process.

\footnotetext{
* Perkin-Elmer stationary phase Z.
} 
For qualitative purposes the temperature may be programmed rapidly, e.g. at $20^{\circ}$ per min from 125 to $275^{\circ}$. For quantitative determinations of MPE and DPE a lower programming rate, e.g. $5^{\circ}$ per min in the range 200 to $230^{\circ}$, is recommended. The injector temperature was held at $300^{\circ}$ and nitrogen $(25 \mathrm{ml}$ per min) was used as carrier gas.

Acknowledgement. This work was supported by a grant from Skånska Ättikfabriken $A B$, Perstorp.

\section{REFERENCES}

1. Kraft, M. J. Chem. Industry (Moscow) 8 (1931) 507.

2. Sporek, K. and Williams, A. F. Analyst 80 (1955) 29.

3. Wyler, J. A. Ind. Eng. Chem., Anal. Ed. 18 (1945) 777.

4. Jaffe, J. H. and Pinchas, S. Anal. Chem. 23 (1951) 1164.

5. Carazzolo, G. Chim. Ind. (Milan) 42 (1960) 858.

6. Wiersma, D. S., Hoyle, R. E. and Rempis, H. Anal. Chem. 34 (1962) 1533.

7. Esposito, G. G. and Swann, M. H. Anal. Chem. 33 (1961) 1855; 34 (1962) 1173.

8. Smith, B. and Carlsson, O. Acta Chem. Scand. 17 (1963) 455.

Received December 29, 1964. 